Zhao, DG

Wang, $Y W^{*}$

Zhou, GL

http://dx.doi.org/10.21278/brod71406

\title{
UNCERTAINTY ANALYSIS OF SHIP MODEL RESISTANCE TEST IN ACTUAL SEAS
}

UDC 629.5.015.2:629.5.018:629.543

Original scientific paper

\section{Summary}

Resistance test is a classical method used to study ship performance. In this study, the uncertainty of large-scale ship model resistance test in actual seas is analyzed. Considering the difference between these trials and traditional test in towing tanks, this study first uses the ITTC 2014 procedure based on GUM to calculate the systematic error in the test. The parameters that affect the test accuracy are also estimated. Then, the program based on the Monte Carlo method is verified, and the differences between the two methods are compared. In this study, the uncertainty sources in the test are quantitatively analyzed, and the results will be helpful for improving the ship model test scheme in actual seas.

Key words: uncertainty; GUM; Monte Carlo method; resistance test; actual seas; large-scale

\section{Introduction}

The resistance test of a ship model in actual seas is conducted using a ship model with a large-scale ratio in a natural water environment. Compared with the towing tank test, this can effectively reduce the influence of the scale effect. In addition, the test environment is similar to the navigation conditions of an actual ship, which can help predict the performance of the ship more accurately [1]. As this technology is still under development and there is very little comparative research data, it is necessary to determine the uncertainty of the test to evaluate the reliability of the test scheme and the validity of the measurement data.

In the course of the experiment, because of the influence of instrument accuracy, data acquisition method, and the environment, it is difficult to obtain true values of the measured physical quantities. In this case, the error range of the test results must be estimated, and uncertainty analysis must be conducted for quantitative analysis in the measurement test. For the ship model test, the ITTC initially followed the American Institute of Aeronautics and Astronautics (AIAA) standard [2], using a set of error analysis methods including bias error and precision error [3]. F Stern et al. [4] provided a summary for AIAA standard for experimental uncertainty assessment. The uncertainty analysis process of single and multiple tests is shown by a simple hydrodynamics experiment. They believed that the benefits of uncertainty assessment in risk far overweigh any actual or perceived time saved in foregoing making estimates. 
With the spread of Guide to Expression of the Uncertainty in Measurement (GUM) [5], many measurement fields regard it as a general rule for evaluating uncertainty. Therefore, the 25th ITTC (2008) suggested that the uncertainty analysis method of the International Standards Organization (ISO), that is, GUM, should be used to evaluate the trust of the experimental measurement results. C DELEN et al. [6] used two ITTC (2002\&2014) procedures based on different standards to analyze the uncertainty of the ship model resistance test in the towing tank. They found that the results obtained by 2002 procedure were higher because it considers more uncertainty sources. While the new procedure greatly simplifies the calculation process, so each has its advantages.

At present, the uncertainty analysis method of resistance test has been gradually improved. Nikolov et al. [7] studied the uncertainty of tow-tank testing of high-speed planning craft and proposed a linear mixed-effect model, which captures both the between-run and within-run variability found in model testing. The proposed method uses all of the time history data to estimate the uncertainty. Therefore, the value of uncertainty obtained is larger than that of the traditional method.

However, whether the procedure is based on the ISO-GUM standard or the AIAA standard, they are essentially the same and can be regarded as they are all based on the TSM (Taylor series method) [8]. To facilitate calculation, these methods ignored the influence of some higher-order terms in the expansion. Therefore, the results may be inaccurate when the uncertainty is large. At the same time, these methods are also difficult to deal with complex models, especially nonlinear models. Therefore, when evaluating the uncertainty of the fullscale maneuverability trials or speed and powering trials, some scholars chose the numerical simulation method based on the Monte Carlo method (MCM) to study the source of uncertainty in the test [9-12]. Zhou et al. [13] used MCM to evaluate the uncertainty of ship model propulsion test in actual seas. They quantified the influence of the environmental parameters and analyzed the composition of the total uncertainty.

In general, since most of the ship model resistance tests are carried out in the towing tank, ITTC procedures can be applied to the evaluation. However, when it comes to the sea trials, the applicability of the procedure is unknown. In this study, two methods will be used to analyze the uncertainty of large-scale ship model resistance test in actual seas. The GUM method is used to evaluate the uncertainty, mainly the influence of systematic error in the test. Then, the reliability of the evaluation is verified by a numerical simulation program based on the MCM. Finally, in view of the contribution of various uncertainty sources in the evaluation results, we put forward some suggestions to improve the test scheme.

\section{Methods}

\subsection{GUM (ITTC 2014)}

According to the Joint Committee for Guides in Metrology (JCGM), uncertainty can be divided into three categories: standard uncertainty, combined uncertainty, and expanded uncertainty.

The value of the standard uncertainty $u$ is equal to the positive square root of the estimated variance. To facilitate the distinction, the GUM method is divided into type A and B according to the different evaluation methods. Type A represents the uncertainty components obtained by the statistical analysis of a series of observed data, and the components obtained by other methods are classified as type B. The analysis content used in this study was the systematic error in the resistance test. The random error in the repetitive test was not considered; therefore, the evaluation method for type B is mainly used. 
The combined standard uncertainty is evaluated according to the transmission of uncertainty, using the following general expression:

$$
u_{c}^{2}(y)=\sum_{i=1}^{N}\left(\partial f / \partial x_{i}\right)^{2} u^{2}\left(x_{i}\right)+2 \sum_{i=1}^{N-1} \sum_{j=i+1}^{N}\left(\partial f / \partial x_{i}\right)\left(\partial f / \partial x_{j}\right) u\left(x_{i}, x_{j}\right)
$$

where $N$ is the number of observed variables. The above expression represents the approximate value of the first-order Taylor expansion of the measurement equation when two or more input quantities are related, that is, are interdependent or correlated. $\partial f / \partial x_{i}$ can be expressed by the sensitivity coefficient $c_{i}$. In the evaluation process used in this study, it is considered that all input quantities are independent. Thus, the equation can be simplified as follows:

$$
u_{c}^{2}(y)=\sum_{i=1}^{N} c_{i}^{2} u^{2}\left(x_{i}\right)
$$

In the application of some industry regulations, it is often necessary to measure uncertainty, that is, to provide an interval that contains most data. The expanded uncertainty can be calculated as

$$
U=k u_{c}(y)
$$

where $k$ is the coverage factor. In general, the value of $k$ is in the range of $2-3$. For the normal distribution function, $k=2$ corresponds to an inclusion probability of approximately $95.45 \%$. In addition, to express the deviation degree of data more clearly, this paper uses the concept of relative uncertainty in the evaluation.

\subsection{Monte Carlo method (MCM)}

The MCM is a supplement for the GUM method proposed by JCGM [14]. It uses a computer to take a large number of samples from the assumed distribution and simulate the test many times to obtain the uncertainty evaluation results. The key is to repeatedly sample the probability density function of the input, $X_{i}$. The implementation steps as follows:

- Set the number of simulations

- Determine the probability density function of the input

- Randomly generate $M$ groups of sample vectors according to the distribution

- Bring each group of vectors into the model equation of the output $Y$ to obtain $M$ values

- Sort the $M$ model values into increasing order to form a new sequence $G$.

- The uncertainty parameters of $Y$ are calculated from $G$, including the best estimated value and standard uncertainty.

\section{Sea Trials}

\subsection{Ship model}

In order to ensure accuracy of the model, we used glass fiber reinforced plastic (GFRP) as the main material of the ship model. Taking the large ore carrier "CSB FORTUNE" as the prototype, we built a 25-meter-long test model. The main parameters are listed in the Table 1. Fig. 1 shows the model hoisting site. 
Table 1 Main parameters of the model

\begin{tabular}{|c|c|c|c|c|c|c|c|c|}
\hline$L_{O A}(\mathrm{~m})$ & $L_{W L}(\mathrm{~m})$ & $L_{p p}(\mathrm{~m})$ & $B(\mathrm{~m})$ & $D(\mathrm{~m})$ & $d(\mathrm{~m})$ & $S\left(\mathrm{~m}^{2}\right)$ & $\nabla\left(\mathrm{m}^{3}\right)$ & $\lambda$ \\
\hline 24.99 & 24.62 & 24.20 & 4.04 & 1.87 & 1.39 & 144.75 & 115.20 & $1: 13$ \\
\hline
\end{tabular}

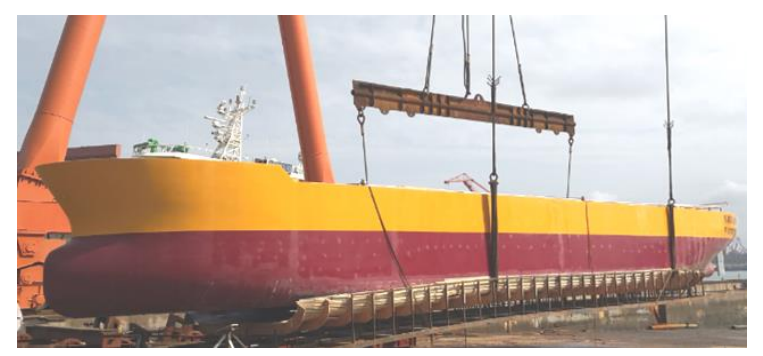

Fig. 1 Ship model

\subsection{Test process}

The test site was in an artificial port in Qingdao, China. To meet the requirements of water conditions set in ISO 15016:2015 [15], the test was carried out when the wind and waves are small (the wave height and wind speed must be lower than $0.2 \mathrm{~m}$ and $3.4 \mathrm{~m} / \mathrm{s}$ ). To reduce the blockage effect of the bottom, the experiment was carried out above half tide conditions. Before the test, the floating state adjustment of the ship model was completed, and the instruments on the ship were debugged.

The implementation of the resistance test is shown in Fig. 2. The two winches in the towing system were installed on the shore at a distance of approximately $300 \mathrm{~m}$. The bow of the ship model was connected with the main traction winch, and the tester adjusted the speed from zero to the specified speed slowly by using the control cabinet, and then uniform traction was maintained. During this process, the tension winch at the tail provides the model with a constant backward friction to help it travel in a straight line. During the test, it was necessary to ensure the position of the force sensor, test cable, and bow and stern lock of the ship model, to avoid sudden and strong damage to the equipment. Simultaneously, to ensure the safety of the crew, equipment, and ship model on board, a safety zone was set up at both sides of the test area; therefore, the uniform speed test distance was approximately $100 \mathrm{~m}$.

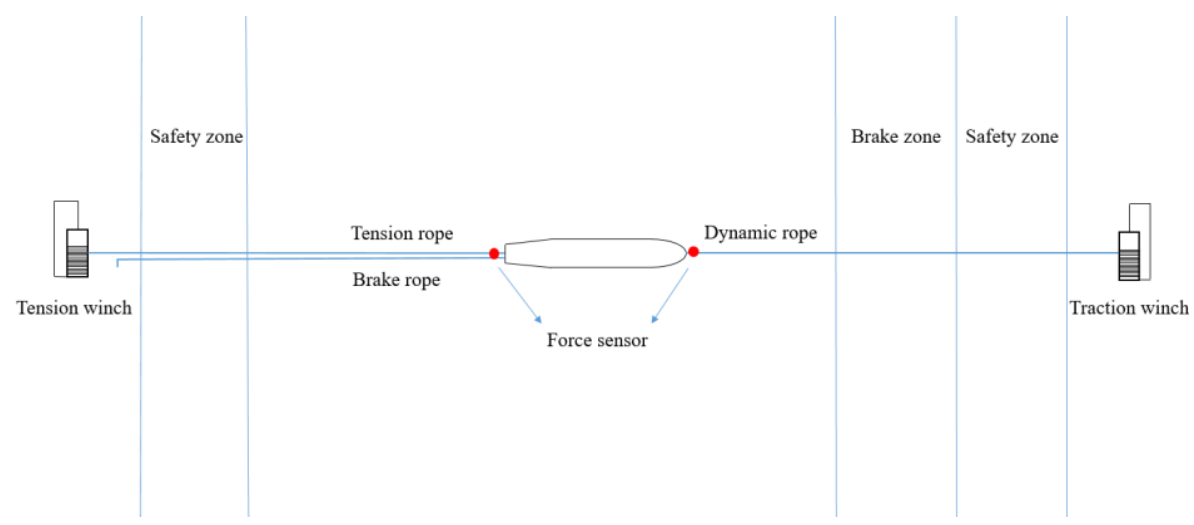

Fig. 2 Schematic of resistance test

Before the test, a small boat and trawler, with a displacement of approximately 30 tons, were tested to verify the reliability of the towing system, as shown in Fig. 3 (a) and (b). The 25$\mathrm{m}$ ship model test is shown in Fig. 3 (c). The different working conditions of the test were realized by adjusting the pulling speed of the cable by using the control cabinet. The actual speed was measured by GPS. 


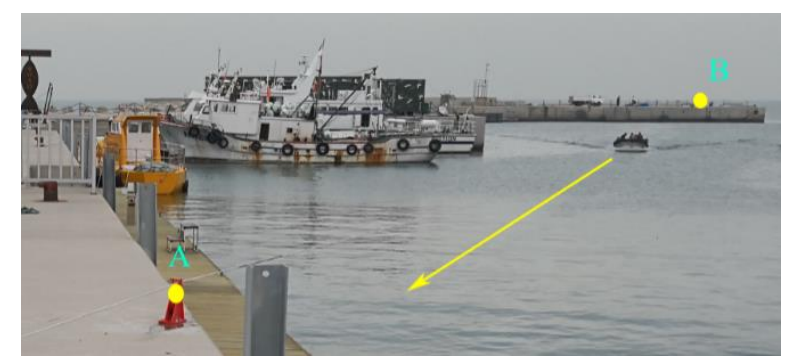

a. Towing test of a boat

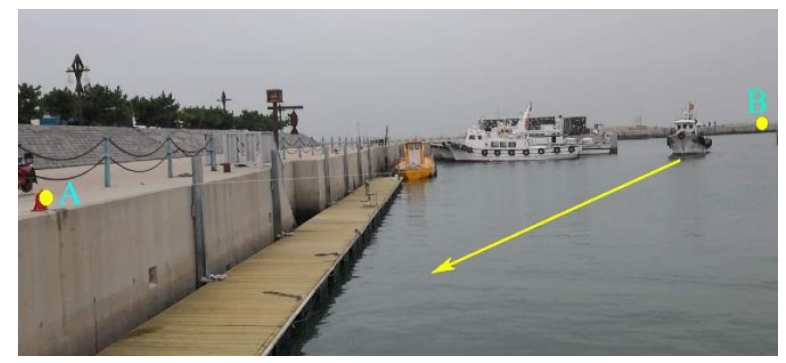

b. Towing test of a trawler

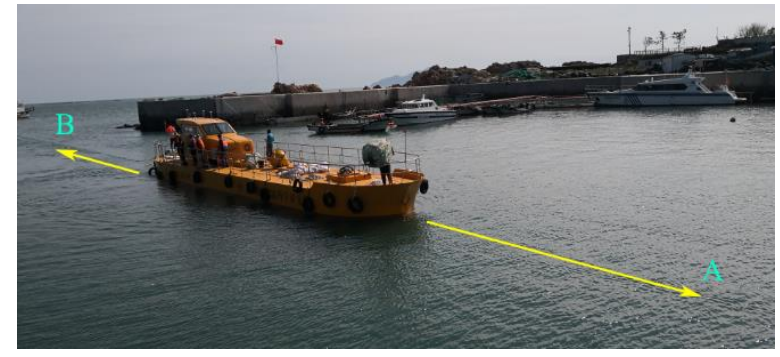

c. Towing test of the ship model

Fig. 3 Resistance test of ship

One resistance test required approximately $0.5 \mathrm{~h}$, considering that the long-term test may introduce interference in the environmental variables. Therefore, for the same working conditions, if the measured data were valid, we only carried out one test.

\section{Results and Discussion}

\subsection{Uncertainty analysis based on GUM method (ITTC 2014)}

The ITTC 2014 procedure [16] calculates the uncertainty of each part relative to the resistance, respectively, and then, obtains the uncertainty of the total resistance coefficient by combining it. In order to improve adaptability, this method ignores some unimportant sources of uncertainty in the evaluation process and simplifies the calculation process. Because no process was repeated in this test, only type B method was used.

\subsubsection{Factors affecting the test}

Referring to the towing tank test procedure recommended by ITTC, the uncertainty sources of resistance test can be divided into the following five parts.

- The uncertainty of the geometric shape of the model mainly arises from the errors produced in the manufacturing process of the ship model, which are mainly caused by the machining accuracy of the workshop. In addition to the length, width, and draught of the ship model, the uncertainty of the wetted surface area is also caused by this error. It is a parameter in the calculation of total resistance coefficient; however, it is 
difficult to obtain directly. Usually, the uncertainty of the wetted surface area can be estimated by the error in ship model displacement.

- The uncertainty sources of test equipment installation include the adjustment of the floating state of the ship model, direction of the dynamometer, towing direction, and course of the model. Because of the stability of the trailer, this effect is usually negligible in pool tests. However, for experiments in actual seas, it is difficult to provide such a stable towing environment; therefore, the corresponding impact can be large.

- All types of instruments on the ship model, including the towing winch, are calibrated before the test. The calibration of the sensor is not discussed in this study, but it was replaced by the precision value given by the manufacturer. In fact, with the improvements in accuracy of electronic instruments, the uncertainty of calibration is negligible for the level of this test.

- The data to be tested in the resistance test include speed, water temperature, resistance, trim, and sinkage. The uncertainty of measurement is reflected in the sampling process of the data points. All data were taken by averaging the measured parameter values. Unlike the towing tank test, the one-way period of this experiment was longer. Considering the real-time variation of the sea environment, we mainly focused on the influence of a systematic error in a single test.

- Finally, the uncertainty of the reduction equation reflects the influence of all factors on the test in the output. The main purpose of this test was to obtain the total resistance coefficient of the ship model, which can be expressed as

$$
C_{T}=R_{T} /\left(\frac{1}{2} \rho S V^{2}\right)
$$

where $S$ is the wetted area of the ship model, $\rho$ is the density of water, $V$ is the towing speed, and $R_{T}$ is the resistance.

\subsubsection{Uncertainty of geometric model}

In sea trials, owing to the influence of waves in the sea and the transverse movement of the model itself, the value of the wetted area cannot be obtained easily. It is difficult to obtain the exact value even in the towing tank test. According to the ITTC procedure, the uncertainty of length and wetted area can be expressed by the displacement of the ship model. According to the form of the dimension, it can be considered that

$$
\begin{aligned}
& S \propto(\nabla)^{2 / 3}, L \propto(\nabla)^{1 / 3} \\
& 2 u^{\prime}(L)=u^{\prime}(S)=\frac{u(S)}{S} \approx \frac{2}{3} u^{\prime}(\nabla)=\frac{2}{3} \frac{\rho_{\text {水 }} u(\nabla)}{\rho_{\text {水 }} \nabla}=\frac{2}{3} u^{\prime}(\Delta)
\end{aligned}
$$

The total resistance of a ship model under a specific Froude number is an equation of the wetted area and Reynolds number. According to the procedure, the changes of resistance with the wetted surface area and length of ship model can be estimated approximately as:

$$
\begin{aligned}
\frac{\delta R_{T}(S)}{R_{T}} & \approx \frac{\partial R_{T}}{\partial S} \cdot \frac{\delta S}{R_{T}}=\frac{\delta S}{S} \\
\frac{\delta R_{T}(L)}{R_{T}} & \approx \frac{\partial R_{T}}{\partial C_{F}} \cdot \frac{\partial C_{F}}{\partial \operatorname{Re}} \cdot \frac{\partial \operatorname{Re}}{\partial L} \cdot \frac{\delta L}{R_{T}} \\
& =\frac{C_{F}}{C_{T}} \cdot \frac{2 / \ln 10}{\log _{10} \operatorname{Re}-2} \cdot \frac{\delta L}{L}
\end{aligned}
$$


So that the influence of the geometric model on resistance can be approximated by the following formula:

$$
\begin{aligned}
u_{11}{ }^{\prime}\left(R_{T}\right) & =u^{\prime}(S) \approx \frac{2}{3} u^{\prime}(\Delta) \\
u_{12}{ }^{\prime}\left(R_{T}\right) & =\frac{C_{F}}{C_{T}} \cdot \frac{0.87}{\lg \operatorname{Re}-2} \cdot u^{\prime}(L) \\
& \approx \frac{C_{F}}{C_{T}} \cdot \frac{0.29}{\lg \operatorname{Re}-2} \cdot u^{\prime}(\Delta)
\end{aligned}
$$

Reynolds number in the test is on the order of $10^{7}$ so that $\mathrm{u}_{12}{ }^{\prime}$ is relatively negligible to $\mathrm{u}_{11}{ }^{\prime}$. When debugging the floating state, the ship model needs to consider the influence of the changes to the model, ballast, equipment, and operators on the total displacement. For this experiment, the deviation of these weights was estimated to be $1000 \mathrm{~kg}$, and the distribution form of the parameter obeys the normal distribution $(k=2)$. It is known that the mass of the ship model is approximately $1.182 \times 10^{5} \mathrm{~kg}$ :

$$
\begin{aligned}
& u(\Delta)=1000 \mathrm{~kg} / 2=500 \mathrm{~kg} \\
& u^{\prime}(\Delta)=500 / 1.182 \times 10^{5}=0.423 \%
\end{aligned}
$$

Therefore, the uncertainty of the geometric model to the resistance is

$$
u_{1}^{\prime}\left(R_{T}\right) \approx \frac{2}{3} u^{\prime}(\Delta)=0.282 \%
$$

\subsubsection{Uncertainty in equipment installation}

This part includes the trim of the ship model, alignment between the model and the midline of the dynamometer, direction of towing, and alignment of the traction force to the transmission bearing. The tests carried out in the towing tank can usually be controlled within high precision in terms of equipment installation; therefore, these effects can often be ignored. However, for the resistance test in actual seas, because both sides of the tension sensor can be moved, and there may be a certain angle between the bow direction and the towing direction of the hoist during the initial towing, this influence is important. It is not easy to obtain accurate values for this uncertainty. The estimated angle here is within the deviation range of $\pm 10^{\circ}$, which is based on the conclusion observed both from the shore and by the operators on board during the test. During the test, the whole test system, including the ship model, ran relatively smoothly, so this estimate can be considered reasonable. The distribution form of this part obeys the normal distribution $(k=2)$. Considering the influence of the angle of the sensors at both sides, the relative uncertainty caused by the installation is

$$
\begin{aligned}
& u_{2}\left(R_{T}\right)=2 \times 580.8 N \times\left(1-\cos 10^{\circ}\right) / 2=8.82 N \\
& u_{2}{ }^{\prime}\left(R_{T}\right)=8.82 / 580.8=1.519 \%
\end{aligned}
$$

\subsubsection{Uncertainty of measurement accuracy}

The uncertainty of instrument accuracy in resistance test mainly arises from three aspects: towing speed, resistance, and water temperature.

The influence of velocity on resistance is in two aspects: the influence of dynamic pressure $\left(1 / 2 \rho V^{2}\right)$ and Reynolds number $(R e)$ : 


$$
\begin{aligned}
\frac{\delta_{1} R_{T}(V)}{R_{T}} & \approx \frac{\partial R_{T}}{\partial\left(\rho V^{2}\right)} \cdot \frac{\partial\left(\rho V^{2}\right)}{\partial V} \cdot \frac{\delta V}{R_{T}}=2 \cdot \frac{\delta V}{V} \\
\frac{\delta_{2} R_{T}(V)}{R_{T}} & \approx \frac{\partial R_{T}}{\partial C_{F}} \cdot \frac{\partial C_{F}}{\partial \operatorname{Re}} \cdot \frac{\partial \operatorname{Re}}{\partial V} \cdot \frac{\partial V}{V} \\
& =\frac{C_{F}}{C_{T}} \cdot \frac{0.87}{\log _{10} \operatorname{Re}-2} \cdot \frac{\partial V}{V}
\end{aligned}
$$

The approximation used here is the same as equation (7). In this way, we can obtain the corresponding uncertainty components in resistance by the following equation:

$$
\begin{aligned}
& u_{41}{ }^{\prime}\left(R_{T}\right)=2 \frac{\delta V}{V}=2 u^{\prime}(V) \\
& u_{42}{ }^{\prime}\left(R_{T}\right)=\frac{C_{F}}{C_{T}} \cdot \frac{0.87}{\lg \operatorname{Re}-2} \cdot u^{\prime}(V)
\end{aligned}
$$

The second term is considerably smaller than the first; therefore, the uncertainty caused by velocity mainly arises from the dynamic pressure.

In this study, the influence of the system error was considered. The uncertainty of towing speed arises from the measurement accuracy of GPS. In this test, it was $0.03 \mathrm{~m} / \mathrm{s}$. It can be considered as a normal distribution and $k=3$. Hence, the uncertainty of velocity is expressed as

$$
\begin{aligned}
& u(V)=0.03 / 3=0.01 \mathrm{~m} / \mathrm{s} \\
& u^{\prime}(V)=0.01 / 1.53=0.654 \%
\end{aligned}
$$

Therefore, the relative standard uncertainty caused by speed is

$$
u_{4}{ }^{\prime}\left(R_{T}\right) \approx 2 u^{\prime}(V)=1.307 \%
$$

The calibration data of the resistance meter are not recorded in this test; thus, the uncertainty caused by the instrument calibration is not calculated in detail. By referring to the data, we can see that the effect of the calibration uncertainty is very small compared with the resistance magnitude of this test. The uncertainty of the resistance balance was calculated using the data given by the HBM manufacturer:

$$
\begin{aligned}
& u_{5}\left(R_{T}\right)=4 N / 3=1.33 N \\
& u_{5}{ }^{\prime}\left(R_{T}\right)=1.33 / 580.8=0.229 \%
\end{aligned}
$$

The influence of water temperature on the test was small, and the influence of density change and model deformation caused by temperature change can be ignored. The main contribution of the water temperature was due to the viscosity coefficient, thus affecting the Reynolds number and friction resistance:

$$
u_{3}^{\prime}\left(R_{T}\right)=\frac{C_{F}}{C_{T}} \cdot \frac{0.87}{\lg \operatorname{Re}-2} \cdot u^{\prime}(v)
$$

The uncertainty of viscosity coefficient can be obtained by reading the deviation of the thermometer, and it obeys uniform distribution.

$$
u^{\prime}(v)=1.93 \% / \sqrt{3}=1.11 \%
$$

Therefore, the relative standard uncertainty caused by water temperature can be expressed as

$$
u_{3}\left(R_{T}\right)=\frac{C_{F}}{C_{T}} \frac{0.87}{\lg \operatorname{Re}-2} u^{\prime}(v)=0.06 \%
$$




\subsubsection{Analysis of uncertainty by GUM}

All the factors affecting the uncertainty of resistance are summarized in Table 2, where the formula for calculating the combined uncertainty is

$$
u_{c}^{\prime}\left(R_{T}\right)=\sqrt{\left(u_{1}{ }^{\prime}\right)^{2}+\left(u_{2}{ }^{\prime}\right)^{2}+\left(u_{3}{ }^{\prime}\right)^{2}+\left(u_{4}{ }^{\prime}\right)^{2}+\left(u_{5}\right)^{2}}
$$

Table 2 Analysis of uncertainty for resistance measurement

\begin{tabular}{|c|c|c|c|}
\hline \multicolumn{4}{|c|}{ Total resistance mean: $R_{T}=580.8 \mathrm{~N}\left(15.6^{\circ} \mathrm{C}\right)$} \\
\hline $\begin{array}{c}\text { Uncertainty } \\
\text { components }\end{array}$ & Type & $\begin{array}{c}\text { Uncertainty, } \\
\text { relative }\end{array}$ & Remark \\
\hline Wetted area & $\mathrm{B}$ & $0.282 \%$ & negligible \\
\hline Towing speed & $\mathrm{B}$ & $1.307 \%$ & minor \\
\hline Water temperature & $\mathrm{B}$ & $0.060 \%$ & negligible \\
\hline Dynamometer & $\mathrm{B}$ & $1.536 \%$ & dominant \\
\hline \multicolumn{2}{|c|}{ Combined uncertainty } & $2.037 \%$ & $u_{C}{ }^{\prime}($ single $)$ \\
\hline \multicolumn{2}{|c|}{ Expanded uncertainty } & $4.075 \%$ & $k=2$ \\
\hline
\end{tabular}

When the water at $15.6{ }^{\circ} \mathrm{C}$ is converted to the nominal temperature $15^{\circ} \mathrm{C}$, we can obtain the resistance expression:

$$
\begin{aligned}
R_{T} & =580.8 N \pm 23.67 N \quad(k=2) \\
& =580.8 N \times(1 \pm 4.075 \%)
\end{aligned}
$$

Therefore, the corresponding total resistance coefficient is obtained as

$$
\begin{aligned}
C_{T} & =3.341 \times 10^{-3} \times(1 \pm 4.075 \%) \quad(k=2) \\
& =3.341 \times 10^{-3} \pm 0.136 \times 10^{-3}
\end{aligned}
$$

The uncertainty of each error source in the test is shown in Fig. 4. It can be seen that the main factor affecting the total resistance coefficient arises from the measurement of velocity and resistance. The error in the towing speed originates from the GPS, which can be improved by improving the measuring accuracy of the instrument. The main problem of the resistance measuring device is the deviation between the towing direction and the course, which can be quantified by designing an angle measuring device. From the overall analysis, increasing the towing speed of the test ship model is helpful in reducing the relative uncertainty of these two aspects. Therefore, without taking into account the cost and size of test sites, an appropriate increase in speed is conducive to improve the accuracy of resistance tests. 


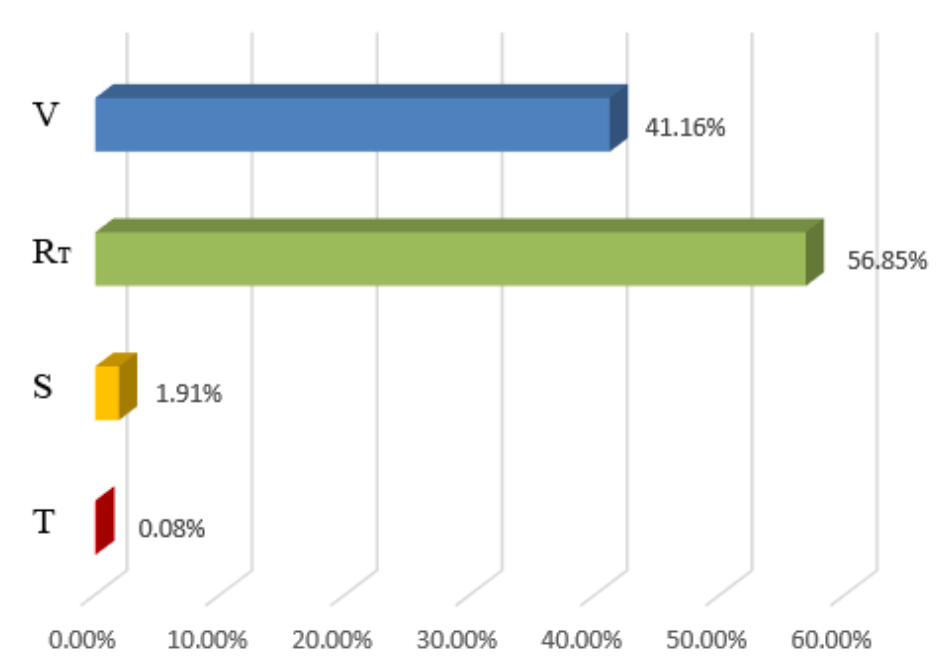

Fig. 4 Uncertainty of each part in the total resistance coefficient

\subsection{Uncertainty analysis based on MCM}

Compared with the evaluation results of the towing tank test, the uncertainty of the resistance test in actual seas was considerably larger. Therefore, to test the reliability of the GUM method for this evaluation, we used MCM.

\subsubsection{Necessity of verification}

In the evaluation process when referring to the ITTC 2014, a certain part is simplified or approximately treated for simplification, which may make the result to be different from the actual value:

- Some relatively small factors are ignored, such as the influence of the captain on the Reynolds number in the geometric model.

- In the evaluation of GUM, the result of total resistance coefficient was approximately equal to the normal distribution. However, by observing the calculation formula and distribution function of several quantities, we can see that the final calculation result should deviate from the standard normal distribution function.

- According to the characteristics of the GUM, the influence of the higher-order term in the Taylor series is not considered in the calculation process.

When the order of uncertainty becomes larger, the impact of these problems on evaluation will increase. To express this deviation trend more clearly, we magnify the uncertainty of the input by ten times to observe the characteristics of the final distribution function. The input parameters are listed in Table 3:

Table 3 Probability distribution of input parameters

\begin{tabular}{|c|c|c|c|}
\hline \multirow{2}{*}{ Input $X_{i}$} & \multirow{2}{*}{ Distribution type } & \multicolumn{2}{|c|}{ Input standard uncertainty $u\left(X_{i}\right)$ scaled $\lambda$ times } \\
\cline { 3 - 4 } & & 1 & 2 \\
\hline$R_{T}(\mathrm{~N})$ & $\mathrm{N}(\mu, \sigma)$ & $\mathrm{N}(580.8,8.92)$ & $\mathrm{N}(580.8,8.92)$ \\
\hline$V(\mathrm{~m} / \mathrm{s})$ & $\mathrm{N}(\mu, \sigma)$ & $\mathrm{N}(1.53,0.01)$ & $\mathrm{N}(1.53,0.10)$ \\
\hline$S\left(\mathrm{~m}^{2}\right)$ & $\mathrm{N}(\mu, \sigma)$ & $\mathrm{N}(144.75,0.41)$ & $\mathrm{N}(144.75,4.10)$ \\
\hline$\rho\left(\mathrm{kg} / \mathrm{m}^{3}\right)$ & $\mathrm{R}(\mathrm{a}, \mathrm{b})$ & $\mathrm{R}(1025.94,1026.10)$ & $\mathrm{R}(1025.21,1026.83)$ \\
\hline
\end{tabular}


Based on the Monte Carlo method, the uncertainty of the total resistance coefficient is simulated by editing the program in MATLAB software. According to the recommendations of the specification, the number of simulations is set to $M=10^{6}$, and the distribution of the output is expressed by frequency histogram, as shown in Fig 5.

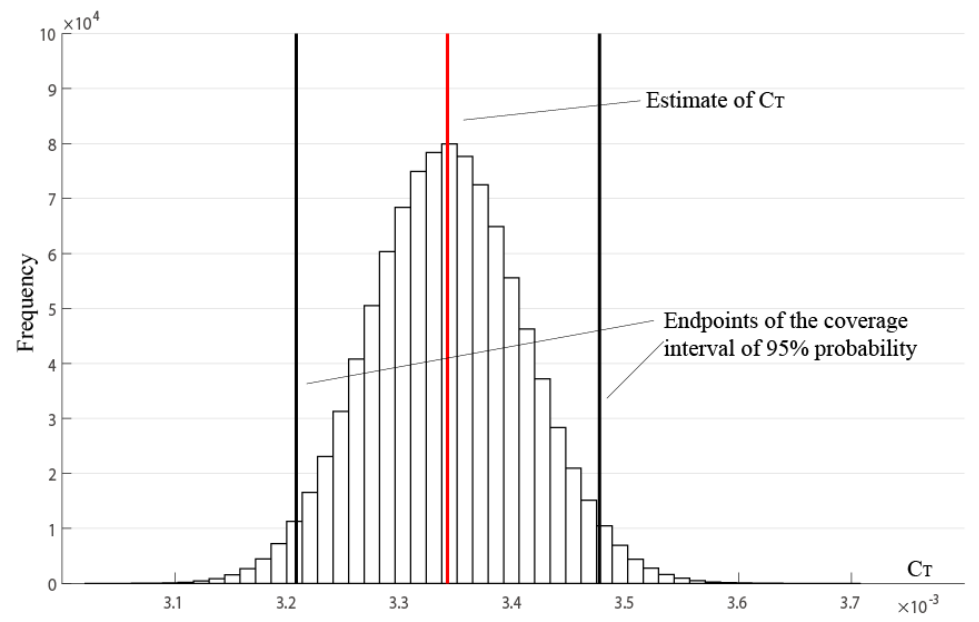

a. $\lambda=1$

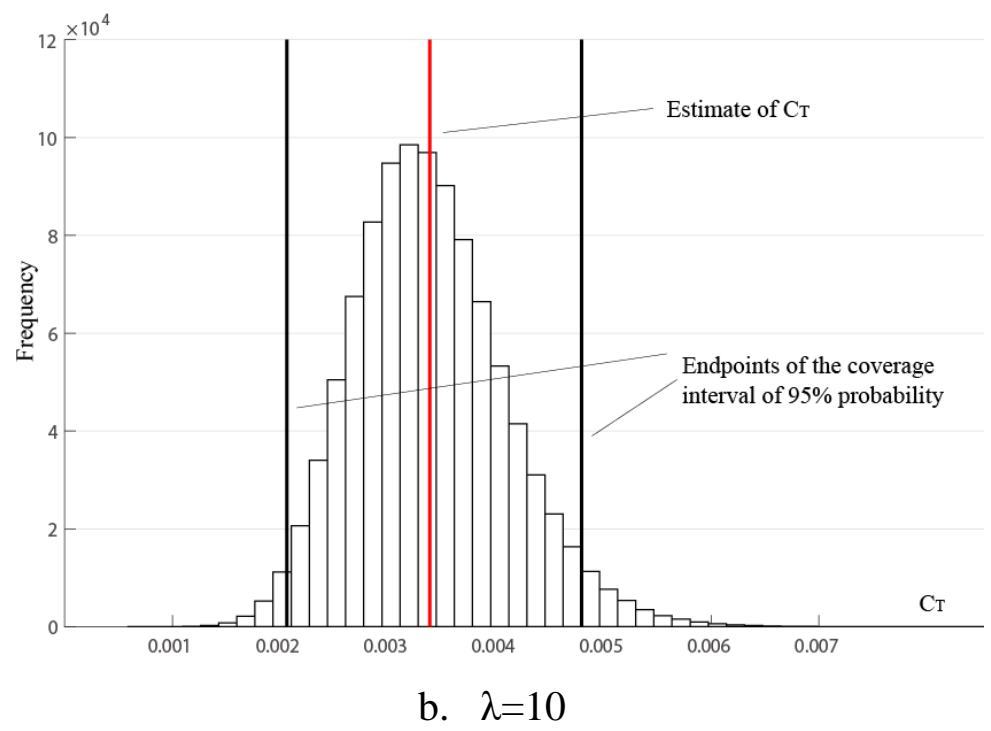

Fig. 5 Distribution frequency of total resistance coefficient under different scaling ratios

Because the group distance of each example is constant in the histograms in Fig. 5 and 6, the frequency (sample number) in a range is directly represented by the height of the rectangle, and the width is the continuous range. The vertical lines represent the average value of the sample, and the upper and lower endpoints of the interval correspond to the $95 \%$ confidence probability. Compared with the frequency distribution in the figure, it is easy to observe that when the uncertainty of the output increases, the deviation between the corresponding probability distribution function and the normal distribution function increases, and the asymmetry becomes more evident. Therefore, it is necessary to use the MCM to verify the evaluation results of GUM.

\subsubsection{Verification results}

The results of uncertainty evaluation of resistance test with GUM are as follows:

$$
u\left(C_{T}\right)=0.068 \times 10^{-3}
$$


The numerical tolerance was set as $u\left(C_{T}\right)$ has 1 significant digit, that is, $n_{\text {dig }}=1$ :

$$
\begin{aligned}
& u\left(C_{T}\right)=7 \times 10^{-5}, c=7, l=-5 \\
& \delta=\frac{1}{2} \cdot 10^{-5}=0.000005
\end{aligned}
$$

To use MCM to verify the results of GUM, the condition is whether the absolute deviation of the end points of the interval obtained by the two methods is less than the numerical tolerance:

$$
\begin{aligned}
& d_{\text {low }}=\left|\left(y-U_{p}\right)-y_{\text {low }}\right| \\
& d_{\text {high }}=\left|\left(y+U_{p}\right)-y_{\text {high }}\right|
\end{aligned}
$$

An adaptive program based on MCM is used, and $M=7.1 \times 10^{5}$ simulations were carried out on the computer. The results are summarized in Table 4.

Table 4 Result of adaptive programs

\begin{tabular}{|c|c|c|c|c|}
\hline$C_{T}\left(10^{-3}\right)$ & $U\left(10^{-3}\right)$ & $d_{\text {low }}\left(10^{-5}\right)$ & $d_{\text {high }}\left(10^{-5}\right)$ & $\delta\left(10^{-5}\right)$ \\
\hline 3.3417 & 0.0681 & 0.28 & 0.21 & 0.5 \\
\hline
\end{tabular}

The absolute deviation of the two terminal values is less than the numerical tolerance; therefore, the GUM is suitable for the uncertainty analysis of this resistance test.

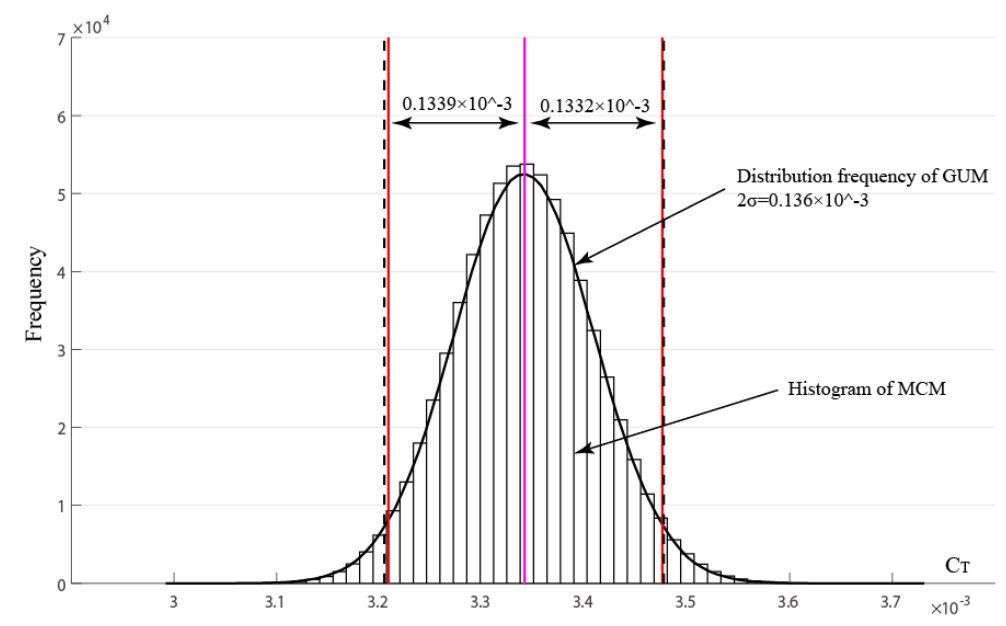

Fig. 6 Comparison of results between GUM and MCM

The probability distribution of the output is shown in Fig 6, where the solid line represents the endpoints of the inclusion interval obtained by the MCM [3.208, 3.475], and the dotted line corresponds to the interval endpoints of the GUM [3.205, 3.477]. The two results are close to each other, in which case the result obtained by the GUM is more conservative, and the value of $C_{T}$ is slightly smaller, which may be because the higher-order terms in the Taylor series was ignored. According to the simulation results of MCM, the total resistance coefficient can be expressed as follows:

$$
\begin{aligned}
C_{T} & =3.3417 \times 10^{-3} \times(1 \pm 4.073 \%) \quad(p=0.95) \\
& =3.3417 \times 10^{-3} \pm 0.1361 \times 10^{-3}
\end{aligned}
$$




\section{Conclusion}

The uncertainty of the test is an index to evaluate the accuracy of the measurement results, and it plays a very important role in the improvement of the test. This paper uses GUM (ITTC 2014) and MCM to evaluate the uncertainty of ship model resistance tests in actual seas. The following conclusions can be drawn:

According to the ITTC 2014 procedure, the relative expanded uncertainty of the total resistance coefficient is $U_{C T^{\prime}}=4.075 \%(k=2)$ based on the GUM. This uncertainty reflects the systematic error in the test.

In the uncertainty transmission of the total resistance coefficient $C_{T}$, the measurement uncertainty of resistance and towing speed are the main sources of error, accounting for $56.9 \%$ and $41.2 \%$, respectively. The former results from the uncertainty of the angle between the towing direction and the navigation direction, while the latter is mainly restricted by the velocity measurement accuracy of the GPS.

Even if the displacement had a large estimated deviation, the effect of the wetted surface area is very small. Therefore, for the resistance test, the measurement accuracy of wetted surface area and water temperature can meet the requirements of the current test.

Due to the less cost for calculation, ITTC 2014 procedure is considered the most commonly used method for resistance test in towing tank. However, it should be noted that in order to simplify the operation, it ignores some uncertainty sources with little effect on tests. Meanwhile, GUM method approximates the higher-order term of Taylor series when calculating the combined standard uncertainty, which indicates that the actual results do not completely conform to the normal distribution. When the input parameter distribution is different and the value is large, the gap becomes apparent, which has been verified in Fig. 5 . Therefore, it is necessary to compare the result with numerical simulation to obtain the range of the deviation.

The program based on the MCM was used to verify the evaluation results of GUM. The results show that the GUM is suitable for the uncertainty analysis of the resistance test in the actual seas. The relative expanded uncertainty of the total resistance coefficient obtained by MCM was $U_{C T^{\prime}}=4.073 \%$. In comparison, the result obtained by the GUM was more conservative, and the total resistance coefficient obtained was smaller.

According to the results of the evaluation, the accuracy of the resistance test can be improved mainly in two aspects: towing speed and resistance measuring device. The uncertainty of towing speed originates from the measuring system, which can start by improving the measurement accuracy of GPS. The main problem of resistance measuring devices is the deviation between the towing direction and the course, which can be quantified by designing a measuring device. In addition, it is easy to find when the speed of the ship model increases, the relative uncertainty about speed will also decrease. Therefore, if the conditions permit, we can consider increasing the towing speed of the ship model to obtain higher test accuracy. Because of the small number of repetitions of the experiment, we mainly studied the systematic error in the experiment. In fact, the random error of the test in the marine environment is a significant part. The next research content will be to shorten the test time of each group and increase the repetition times and stability of the test. Then, the uncertainty related to the data fluctuation in the test process can be studied. Compared with the towing tank tests, the uncertainty of the resistance test in actual seas is much larger. Although the results of our work may be helpful to improve the test accuracy, it is very difficult to reach the magnitude of the traditional experiment. This is partly because sea trials do not have such stable test system like tests in towing tank. On the other hand, the influence of marine environment on the test is also enormous. At present, taking resistance tests in towing tank is still the main experimental 
method to analyze ship performance. But we believe that such tests in actual sea, as a new experimental technology, will have a broader application in the future.

\section{Acknowledgement}

This work was supported by the National Natural Science Foundation of China (grant number 51709060)

\section{REFERENCES}

[1] Jiao, J., Sun, S., \& Ren, H. (2016). Predictions of wave induced ship motions and loads by large-scale model measurement at sea and numerical analysis. Brodogradnja: Teorija i praksa brodogradnje i pomorske tehnike , 67 (2), 81-100. https://doi.org/10.21278/brod67206

[2] AIAA (1999) S-071A, 1999, Assessment of Experimental Uncertainty with Application to Wind Tunnel Testing, American Institute of Aeronautics and Astronautics.

[3] ITTC (1999) Uncertainty Analysis in EFD, Uncertainty Assessment Methodology, ITTC Recommended Procedures and Guidelines 7.5-02-01-01, Revision 00.

[4] Stern, F., Muste, M., Beninati, M. L., \& Eichinger, W. E. (1999). Summary of experimental uncertainty assessment methodology with example (No. 406). IIHR Report.

[5] JCGM, J. (2008). Evaluation of measurement data - Guide to the expression of uncertainty in measurement. Int. Organ. Stand. Geneva ISBN, 50, 134.

[6] DELEN, C., \& Şakir, B. A. L. (2015). Uncertainty analysis of resistance tests in Ata Nutku Ship model testing Laboratory of Istanbul Technical University. Türk Denizcilik ve Deniz Bilimleri Dergisi, 1(2), 6988. https://doi.org/10.1201/b18855-7

[7] Nikolov, M. C., \& Judge, C. Q. (2017). Uncertainty Analysis for Calm Water Tow Tank Measurements. Journal of Ship Research, 61(4), 177-197. https://doi.org/10.5957/JOSR.160072

[8] Coleman, H. W., \& Steele, W. G. (2018). Experimentation, validation, and uncertainty analysis for engineers. John Wiley \& Sons. https://doi.org/10.1002/9781119417989

[9] Insel, M. (2008). Uncertainty in the analysis of speed and powering trials. Ocean Engineering, 35(11-12), 1183-1193. https://doi.org/10.1016/j.oceaneng.2008.04.009

[10] Woodward, M. D. (2014). Evaluation of inter-facility uncertainty for ship manoeuvring performance prediction. Ocean engineering, 88, 598-606. https://doi.org/10.1016/j.oceaneng.2014.04.001

[11] Gavrilin, S., \& Steen, S. (2017). Uncertainty of full-scale manoeuvring trial results estimated using a simulation model. Applied Ocean Research, 64, 281-289. https://doi.org/10.1016/j.apor.2017.03.011

[12] Seo, D. W., Kim, M. S., \& Kim, S. Y. (2019). Uncertainty Analysis for Speed and Power Performance in Sea Trial using Monte Carlo Simulation. Journal of the Society of Naval Architects of Korea, 56(3), 242250. https://doi.org/10.3744/SNAK.2019.56.3.242

[13] Zhou G, Wang Y, Zhao D, Lin J (2020). Uncertainty Analysis of Ship Model Propulsion Test on Actual Seas Based on Monte Carlo Method. Journal of Marine Science and Engineering, 8(6):398. https://doi.org/10.3390/jmse8060398

[14] JCGM, Y. (2008). Evaluation of measurement data-Supplement 1 to the guide to the expression of uncertainty in measurement-Propagation of distributions using a Monte Carlo method. Organization for Standardization (Geneva, Switzerland).

[15] ISO 15016 (2015). Marine Technology - Guidelines for the assessment of speed and power performance by analysis of speed trial data.

[16] ITTC (2014) General Guideline for Uncertainty Analysis in Resistance Tests, ITTC Recommended Procedures and Guidelines 7.5-02-02-02, Revision 02.

Submitted: $\quad$ 21.02.2020. Zhao, DG, College of Shipbuilding Engineering, Harbin Engineering

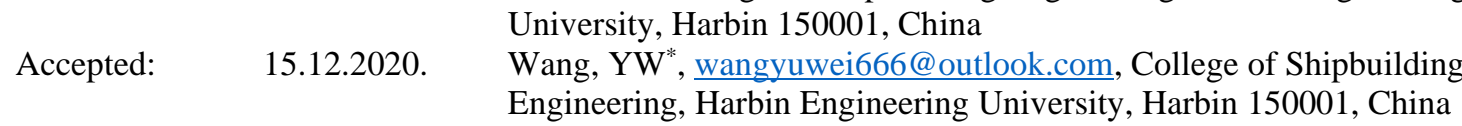
Zhou, GL, College of Shipbuilding Engineering, Harbin Engineering University, Harbin 150001, China 